\title{
Proximity Effect in Periodic Arrays of Superconducting Nanoislands on Thin Graphite Layer
}

\author{
Yuri Latyshev $^{1,2}$, Anatoly Smolovich ${ }^{1, ~}$, Andrey Orlov ${ }^{1,2}$, Aleksei Frolov ${ }^{1,3}$, Vyacheslav Vlasenko ${ }^{4}$ \\ ${ }^{1}$ Kotel'nikov Institute of Radio Engineering and Electronics of the RAS, Russia \\ ${ }^{2}$ Laboratoire National des Champs Magnétiques Intenses, France \\ ${ }^{3}$ Moscow Institute of Physics and Technology, State University, Russia \\ ${ }^{4}$ OPTEC LLC, Russia
}

Copyright $(\subset 2015$ by authors, all rights reserved. Authors agree that this article remains permanently open access under the terms of the Creative Commons Attribution License 4.0 International License

\begin{abstract}
The regular structure of superconducting nanoislands of alloy $\mathrm{W}-\mathrm{Ga}-\mathrm{C}$ was fabricated on nanothin graphite using focused ion beam. The resistance vs temperature dependence down to $1.7 \mathrm{~K}$ and the magnetoresistance in field up to $24 \mathrm{~T}$ were measured both for the bridge containing nanoislands and for the reference bridge without islands. The difference between those measurements demonstrates the proximity effect on a regular structure of superconducting W-Ga-C nanoislands on nanothin graphite layer.
\end{abstract}

Keywords Graphene, Dirac Fermions, Induced Superconductivity, Focused Ion Beam Deposition, Proximity Effect

\section{Introduction}

Graphene is a material which has an unique electronic spectrum. Carriers in graphene are massless chiral Dirac fermions. Since the discovery of graphene in 2004 [1] electronic structure of graphene was studied in detail [2-4]. Today hybrid systems based on graphene are of great interest. In such systems the interaction of Dirac fermions with other quasiparticles significantly changes properties of the system. In several papers it was studied the interaction between graphene and various systems: superconductors, thin films (2D) [5], nanowires (1D) [6], nanoholes and nanoperforations (0D) [7-9] and others. The authors of paper [10] considered theoretically a hybrid structure of superconducting islands array placed at the surface of graphene. A strong collective proximity effect in this structure was predicted due to a high mobility of massless carriers in graphene - Dirac fermions. Thus, superconductivity in graphene spreads at macroscopic lengths.

There were several experimental studies of the Josephson current through graphene in standard wide planar SNS junctions $[11,12]$. However, until now experimental studies investigating this effect on the regular array structures are practically absent. There were only a few papers on studies this effect in non-regular structures [13]. Recently the first paper on studies of regular structure on graphene has been appeared [14]. Proximity effect in a system consisting of gold thin-films covered with an array of niobium nano-discs was studied in [15].

We report here on fabrication and investigation of the regular structure of superconducting islands on nanothin graphite. It is known that the surface of nanothin graphite also demonstrates the properties of Dirac fermions [8, 16-18]. The STM [19], cyclotron resonance [20] and Raman spectroscopy $[21,22]$ experiments showed that the surface layer of graphite is often represented as a graphene layer of an exceptional quality. In such system the Dirac fermions scattering is considerably lower than even in suspended graphene sheets. This graphene-on-graphite system is the most attractive for thin enough graphite samples to avoid the shunting of the surface graphene layers by the bulk

\section{Materials and Methods}

We obtained films of nanothin graphite using following technique: high-quality crystals of natural graphite were cleaned and then attached to the substrate using epoxy glue and then thinned down to tens of nanometers by adhesive tape $[23,24]$. For making the surface of graphene clean we simply removed higher layers of graphite using adhesive tape. The flatness of the surface was controlled by optical microscope Carl Zeiss Axio Imager (with maximum magnification $\mathrm{x} 1000)$ in differential interference contrast (DIC) regime. Usually we used 2-component 5-min epoxy glue UHU Schnellfest, which has excellent adhesive properties and flat surface without defects after drying. We usually used polycor $\left(\mathrm{Al}_{2} \mathrm{O}_{3}\right)$ or silicon with 300-nm $\mathrm{SiO}_{2}$ layer as a substrate. After thinning we were looking for a 
suitable graphite films (atomic thicknesses, dimensions more than $0.3 \times 0.3 \mathrm{~mm}$, with flat surface) using optical microscope in transmitting and reflecting modes. We controlled thicknesses of graphite films by light transmittance. It is known [25] that one graphene layer absorbs $2.3 \%$ of light independent of wavelength. We used translucent graphite films corresponding to thicknesses lower than $50 \mathrm{~nm}$. The excess of films was cleaned off by razor blade.

After the suitable atomically thin graphite film was prepared we fabricated ohmic contacts to the sample. Graphite film was partially masked by whisker ribbons. Then Au contacts were manufactured on the laboratory laser ablation setup. The setup used the third harmonic $(355 \mathrm{~nm})$ of Nd: YAG laser with $0.2 \mathrm{~J}$ pulse energy, $10 \mathrm{~ns}$ pulse duration, and $6 \mathrm{~Hz}$ pulse repetition. Then the whisker mask was removed.

We tried to produce superconducting nanoislands on thin graphite by local tungsten (W) deposition using focused electron and ion beams of CrossBeam EsB 1540 (Carl Zeiss). We obtained the minimal diameter of nanoislands about 10 $\mathrm{nm}$ for electron beam deposition and 60-80 $\mathrm{nm}$ for ion beam deposition. However, we chose the ion beam deposition for our experiment because it was known from the literature [26-30] that in this case an alloy W-Ga-C is actually deposited. The alloy W-Ga-C temperature of superconducting transition $T_{c}$ is about $4-5 \mathrm{~K}$ that is much higher than $T_{c}$ of pure $W$. We produced the arrays from $10 \times 7$ to $40 \times 20$ nanoislands with the spacing from 100 to $300 \mathrm{~nm}$ (Fig. 1). For $\mathrm{T}_{\mathrm{c}}$ measurement $\mathrm{W}-\mathrm{Ga}-\mathrm{C}$ band was deposited. The measured resistance vs temperature dependence for W-Ga-C band is plotted on Insert of Fig. 2. We obtained $T_{c}$ $\approx 5 \mathrm{~K}$. To provide comparable measurements we fabricated two similar bridges, one containing W-Ga-C nanoislands (Fig.2) and another - without them (reference sample).

The measurements in magnetic field (up to $23.5 \mathrm{~T}$ ) were performed with $10 \mathrm{MW}$ Bitter magnet in the helium cryostat with possibility of cooling down to $1.7 \mathrm{~K}$ in Laboratoire National des Champs Magnétiques Intenses, Grenoble, France. The magnetic field was changed with rate 1-2 $\mathrm{T} / \mathrm{min}$. The samples resistance was measured by $\mathrm{DC}$ Current Reversal Method (with current value from $10 \mathrm{nA}$ till $10 \mathrm{uA}$ ) for thermoelectric EMFs excluding. Keithley SourceMeter 2636A (with resolution of $20 \mathrm{fA}$ ) was used as a current source. Two nanovoltmeters Agilent 34420A (with resolution of $0.1 \mathrm{nV}$ ) were used for the synchronous voltage measurements on the sample containing W-Ga-C nanoislands and on the reference sample.

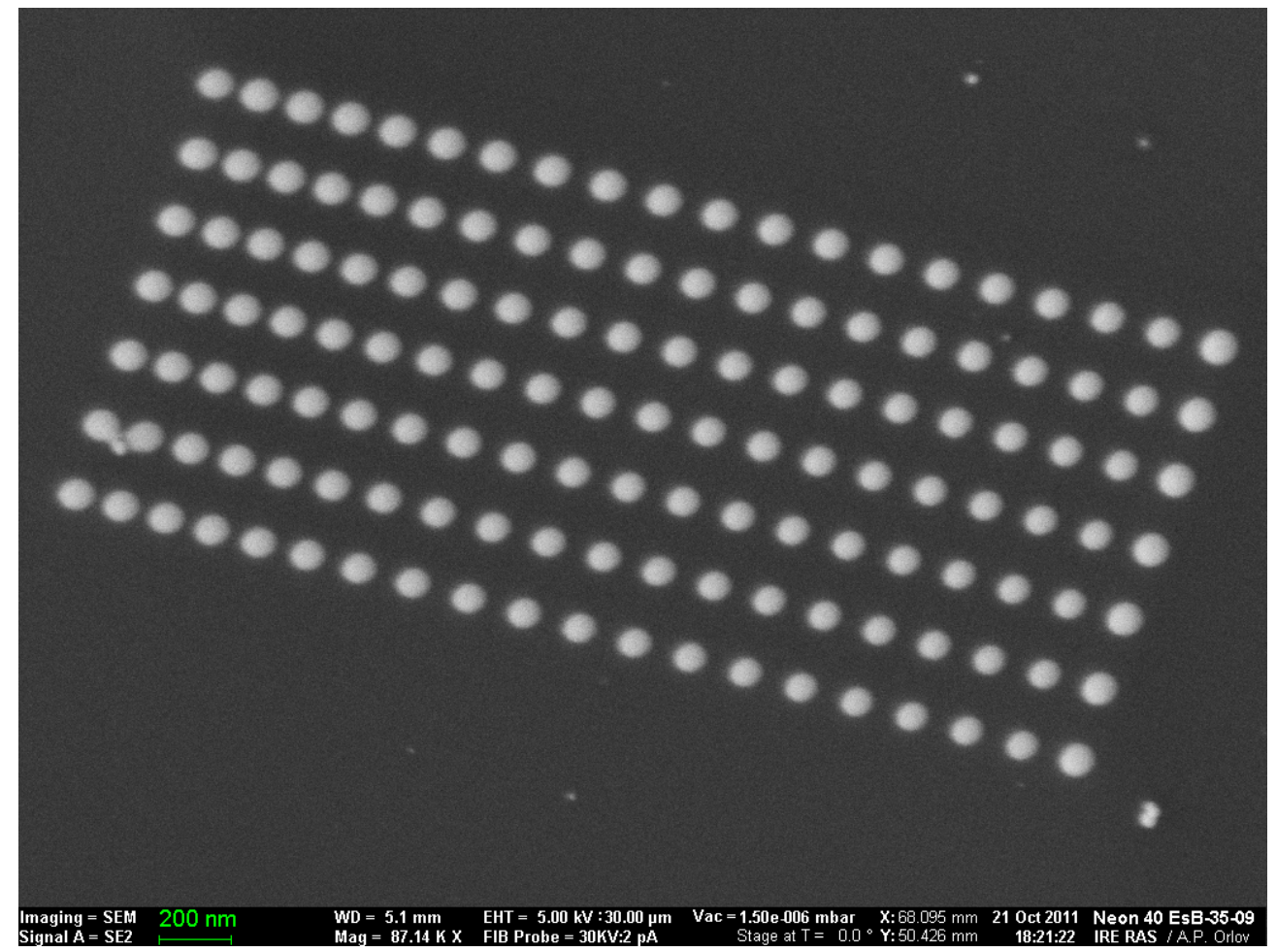

Figure 1. W-Ga-C nanoislands array on thin graphite. Nanoisland diameter is about $90 \mathrm{~nm}$. Array spacing is $160 \mathrm{~nm}$ in horizontal row and $210 \mathrm{~nm}$ in vertical row. 


\section{Results and Discussion}

Temperature dependences of the electro-resistance R (T) for both samples have similar character down to $5 \mathrm{~K}$, whereas below $5 \mathrm{~K}$ the resistance of sample with nanoislands goes up considerably flatter than the resistance of reference bridge (Fig.3) indicating a proximity effect between the islands. Note, that the resistance of the sample with nanoislands is much higher than the resistance of the reference sample due to the sample damage by ion beam [31] during the deposition process. So, we compare the shapes of the corresponding $\mathrm{R}(\mathrm{T})$ curves and not the absolute values of the sample resistance.

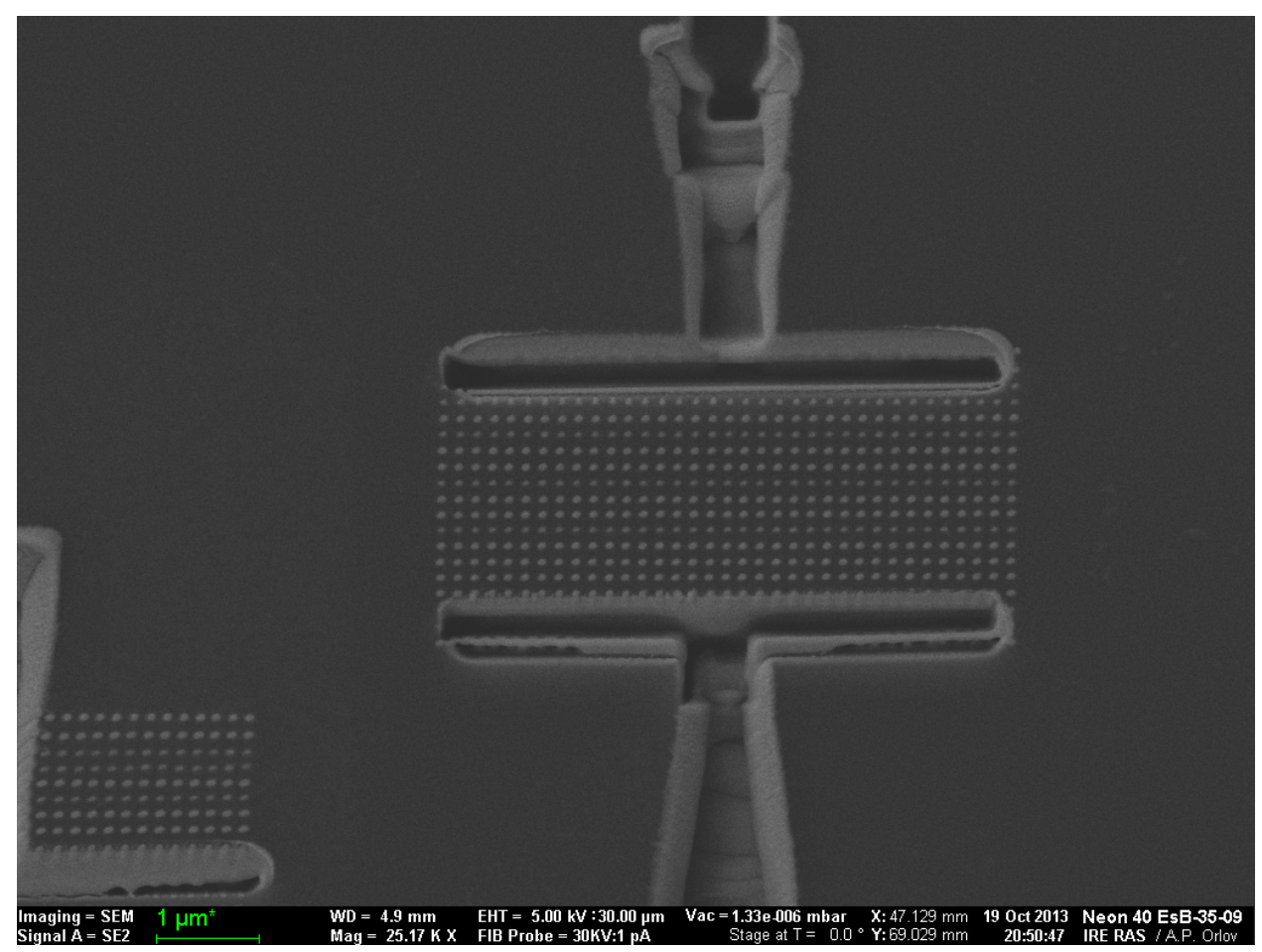

Figure 2. Graphite bridge with W-Ga-C nanoislands.

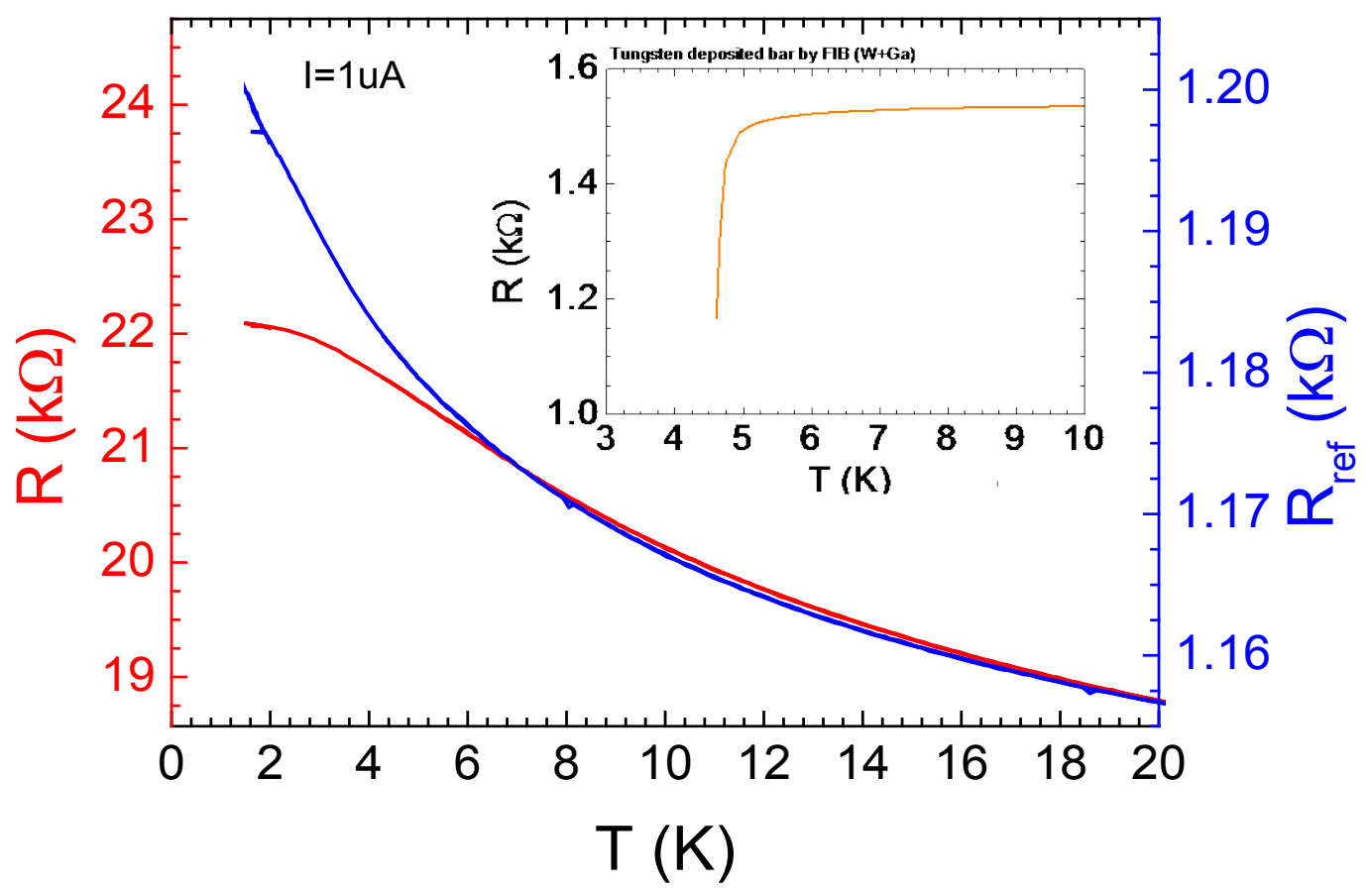

Figure 3. The resistance vs temperature dependences for samples containing the array of W-Ga-C nanoislands (red curve) and for reference sample without nanoislands (blue curve). The island diameter is $90 \mathrm{~nm}$ and the grating period is $170 \mathrm{~nm}$. The lateral sizes of both bridges are $3 \times 1.3 \mathrm{um}$. Inset: superconducting junction at the temperature dependence for W-Ga-C band. 


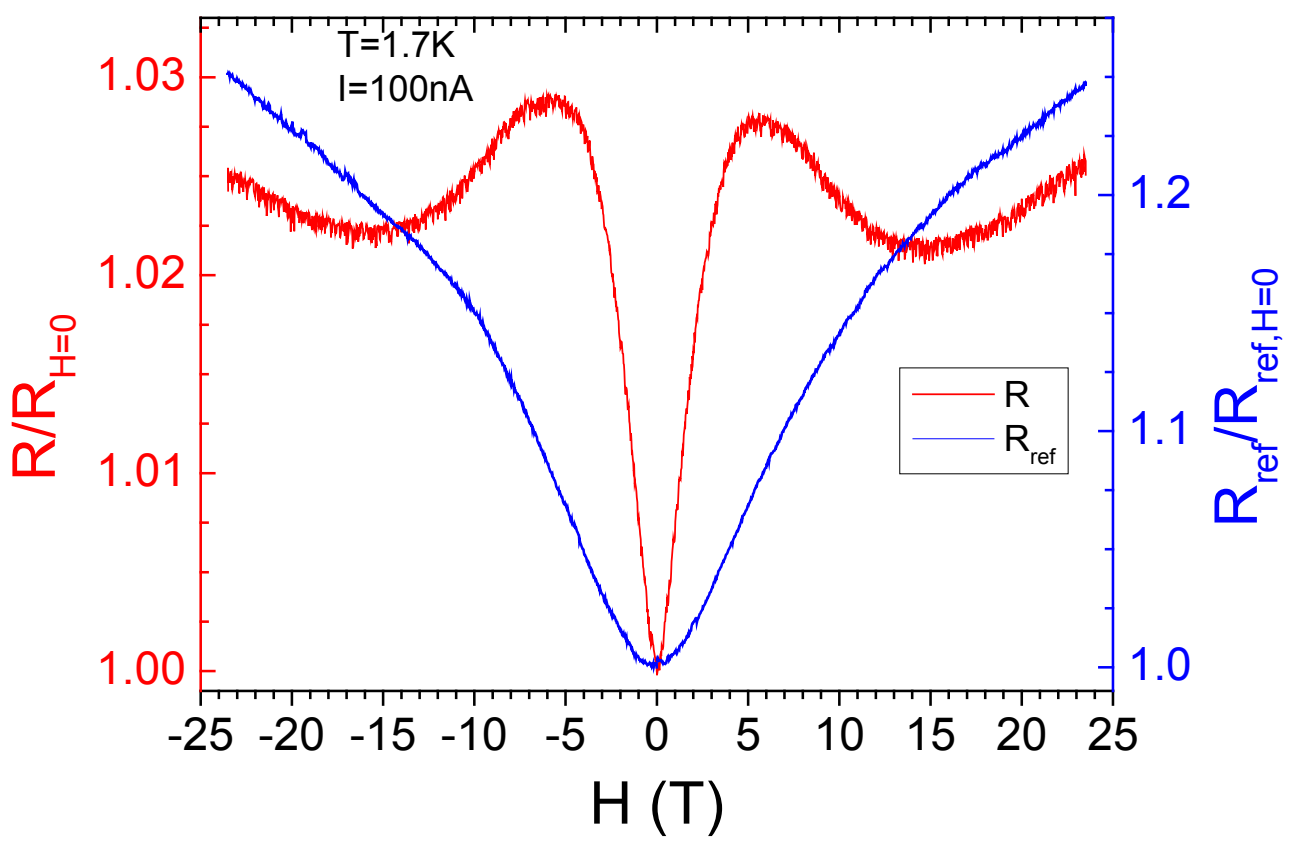

Figure 4. The magnetoresistance of the graphite bridge with superconducting nanoislands (red curve) and the reference bridge (blue curve).

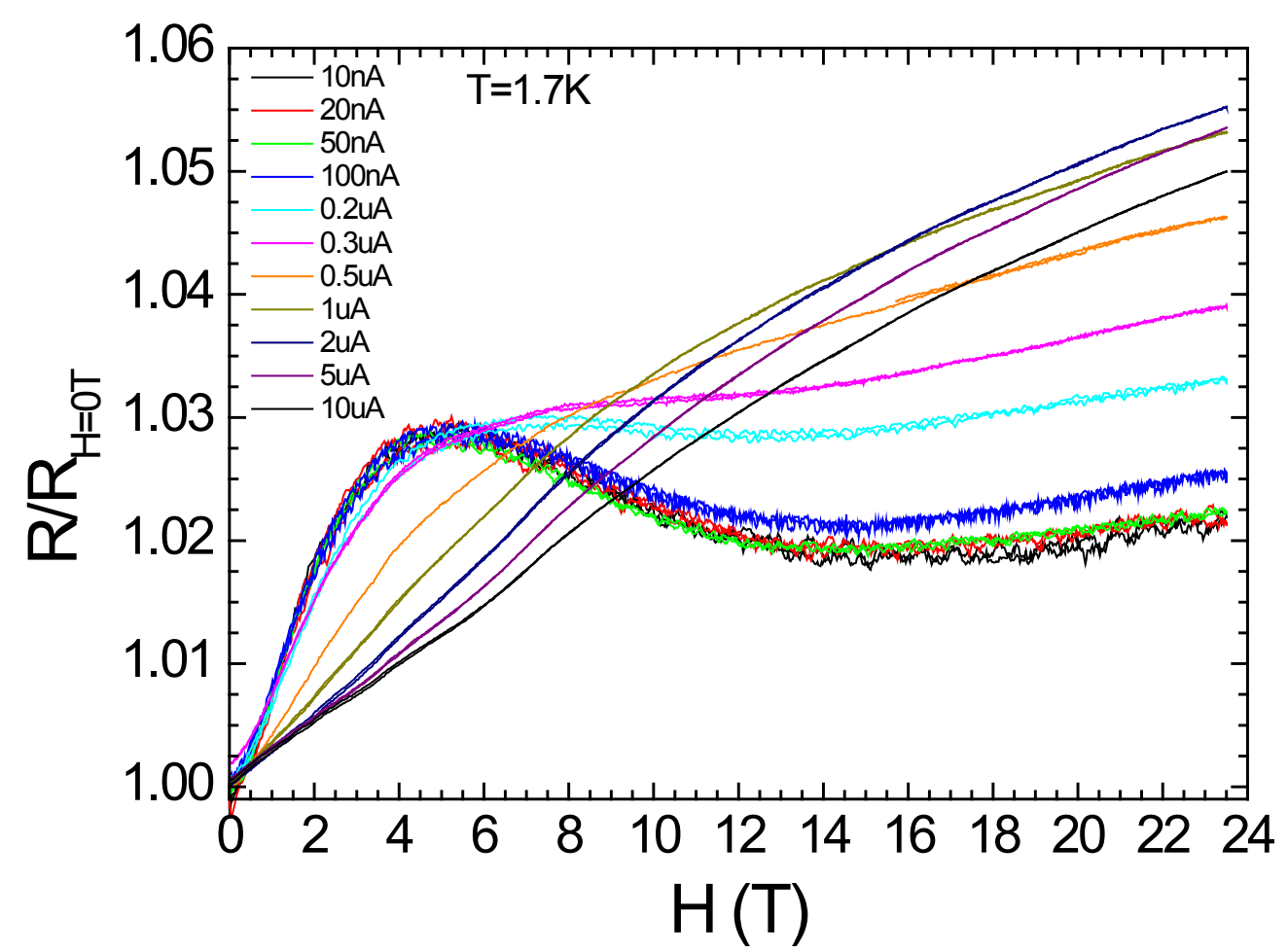

Figure 5. The magnetoresistance of the graphite bridge with superconducting nanoislands for different probe currents.

Accordingly, the dependence of $\mathrm{R}(\mathrm{H})$ of the sample with islands first increases rapidly, which we attribute to the suppression of weak coupling between the islands, then proceeds to a negative magnetoresistance region, and finally goes to the usual dependence of $\mathrm{R}(\mathrm{H})$ similar to a reference sample (Fig.4). A region of negative magnetoresistance is attributed to the destruction of superconductivity in nanoislands at fields $\mathrm{H}_{\mathrm{c} 1}<\mathrm{H}<\mathrm{H}_{\mathrm{c} 2}$ with a corresponding redistribution of the field and the weakening of its value between the islands, which leads to a decrease in the total sample magnetoresistance. Additionally, the suppression of superconductivity of nanoislands is also accomplished by an increase of normal carries, which become non-localized inside the island and contribute to total conductivity. A region of negative magnetoresistance has also been observed on the structures with irregular superconducting islands on graphene [13]. The increase of current through the sample leads to vanishing of a negative magnetoresistance region (Fig.5). At currents higher than of $500 \mathrm{nA}$ the magnetoresistance of the graphite bridge with 
superconducting nanoislands looks similar to the magnetoresistance of the reference bridge. Also, the difference in behavior between the graphite sample with superconducting nanoislands and the reference sample vanished if the graphite thickness was increased up to one hundred of graphene monolayers.

\section{Conclusions}

Graphene with superconductive nanoislands was studied. The periodic array of nanoislands of alloy $\mathrm{W}-\mathrm{Ga}-\mathrm{C}$ was fabricated on nanothin graphite by focused ion beam deposition. The nanoislands diameter was about $80 \mathrm{~nm}$ and the array spacing was from 100 to $300 \mathrm{~nm}$. The resistance vs temperature dependence down to $1.7 \mathrm{~K}$ and the magnetoresistance in field up to $24 \mathrm{~T}$ were measured both for the bridge containing nanoislands and for the reference bridge without islands. The resistance vs temperature curve below $5 \mathrm{~K}$ goes up considerably flatter for the bridge containing nanoislands. The resistance vs magnetic field curve shape for the bridge containing nanoislands differs considerably from the one for the bridge without nanoislands and has a region of negative magnetoresistance. Both differences were explained as the display of the proximity effect on the sample with superconductive nanoislands.

\section{Acknowledgements}

The first author, Prof. Yuri Latyshev passed away in Moscow on 10th June 2014.

The authors thank A.A. Sinchenko for helpful discussions and V.A. Shakhunov for sample contacts manufacturing. The work has been supported by RFBR (grants No. 14-02-01126-a, 14-02-01166-a and 13-02-00927-a), by the programs of RAS, and by the Laboratoire National des Champs Magnétiques Intenses (Grenoble).

The work was presented on the 4th International scientific conference state-of-the-art trends of scientific research of artificial and natural nanoobjects (STRANN '14) 22-25, April 2014 Saint-Petersburg, Russia [32].

\section{REFERENCES}

[1] K.S. Novoselov, A.K. Geim, S.V. Morozov, D. Jiang, Y. Zhang, S.V. Dubonos, I.V. Grigorieva, A.A. Firsov, Electric Field Effect in Atomically Thin Carbon Films, Science 306, 666-669 (2004).

[2] A. H. Castro Neto, F. Guinea, N. M. R. Peres, K. S. Novoselov, A. K. Geim, The electronic properties of graphene, Rev. Mod. Phys. 81, 109-162 (2009).

[3] Geim, A. K., Novoselov, K. S., The rise of graphene, Nature Materials 6 (3), 183-91 (2007).

[4] S. Das Sarma, S. Adam, E. H. Hwang, and E. Rossi,
Electronic transport in two-dimensional graphene, Rev. Mod. Phys. 83, 407-470 (2011).

[5] Sung Won Hwang, Dong Hee Shin, Chang Oh Kim, Seung Hui Hong, Min Choul Kim, Jungkil Kim, Keun Yong Lim, Sung Kim, Suk-Ho Choi, Kwang Jun Ahn, Gunn Kim, Sung Hyun Sim, and Byung Hee Hong, Plasmon-Enhanced Ultraviolet Photoluminescence from Hybrid Structures of Graphene/ZnO Films, Phys. Rev. Lett. 105, Article number: 127403 (2010).

[6] Mi-Sun Lee, Kyongsoo Lee, So-Yun Kim, Heejoo Lee, Jihun Park, Kwang-Hyuk Choi, Han-Ki Kim, Dae-Gon Kim, Dae-Young Lee, SungWoo Nam, and Jang-Ung Park, High-Performance, Transparent, and Stretchable Electrodes Using Graphene-Metal Nanowire Hybrid Structures, Nano Letters 13 (6), 2814-2821 (2013).

[7] Yu. I. Latyshev, A. P. Orlov, A. V. Frolov, V. A. Volkov, I.V.Zagorodnev, V.A. Skuratov, Yu.V. Petrov, O.F. Vyvenko, D.Yu. Ivanov, M. Konczykowski, P. Monceau, Orbital quantization in a system of edge Dirac fermions in nanoperforated graphene, Journal of Experimental and Theoretical Physics Letters, 98, 214-218 (2013).

[8] Yu.I. Latyshev, A.Yu. Latyshev, A.P. Orlov, A.A. Schekin, V.A. Bykov, P. Monceau, C. van der Beek, M. Konczykowski, I. Monnet, Field-periodic magnetoresistance oscillations in thin graphite single crystals with columnar defects, Journal of Experimental and Theoretical Physics Letters, 90, 480-484 (2009).

[9] Yu I. Latyshev, A. P. Orlov, V. A. Volkov, V.V. Enaldiev, I.V. Zagorodnev, O. F. Vyvenko, Yu V. Petrov, P. Monceau, Transport of Massless Dirac Fermions in Non-topological Type Edge States, Scientific Reports 4, Article number: 7578 (2014).

[10] M.V. Feigel'man, M.A. Skvortsov, K.S. Tikhonov, Proximity-induced superconductivity in graphene, Journal of Experimental and Theoretical Physics Letters, 88, 747-751 (2008).

[11] H.B. Heersche, P. Jarillo-Herrero, J.B. Oostinga, L.M.K. Vandersypen, and A.F.Morpurgo, Bipolar Supercurrent in Graphene, Nature 446, 56-59 (2007).

[12] Xu Du, Ivan Skachko, and Eva Y. Andrei, Josephson current and multiple Andreev reflections in graphene SNS junctions, Phys. Rev. B 77, Article number: 184507 (2008).

[13] A. Allain, Z. Han, V. Bouchiat, Electrical control of the superconducting-to-insulating transition in graphene-metal hybrids, Nature Mater. 11, 590-594 (2012).

[14] Zheng Han, Adrien Allain, Hadi Arjmandi-Tash, Konstantin Tikhonov, Mikhail Feigel'man, Benjamin Sacépé, Vincent Bouchiat, Collapse of superconductivity in a hybrid tingraphene Josephson junction array, Nature Physics 10, 380386 (2014).

[15] S. Eley, S. Gopalakrishnan, P.M. Goldbart, N. Mason, Approaching zero-temperature metallic states in mesoscopic superconductor_normal_superconductor arrays, Nature Phys. 8, 59-62 (2012).

[16] M. Orlita, C. Faugeras, G. Martinez, D. K. Maude, M. L. Sadowski, and M. Potemski, Dirac Fermions at the H Point of Graphite: Magnetotransmission Studies, Phys. Rev. Lett. 100, Article number: 136403 (2008). 
[17] Guohong Li and Eva Y. Andrei, Observation of Landau levels of Dirac fermions in graphite, Nature Physics 3, 623-627 (2007).

[18] Yu.I. Latyshev, Z.Ya. Kosakovskaya, A.P. Orlov, A.Yu. Latyshev, V.V. Kolesov, P. Monceau, and D. Vignolles, Nonlinear interlayer transport in the aligned carbon nanotube films and graphite, Proceedings of the International Conference on Theoretical Physics 'Dubna-Nano 2008'. Journal of Physics: Conference Series 129, Article Number: 012032 (2008)

[19] G. Li, N. Luicann, and E. Y. Andrei, Scanning Tunneling Spectroscopy of Graphene on Graphite. Phys. Rev. Lett. 102, Article Number: 176804 (2009).

[20] P. Neugebauer, M. Orlita, C. Faugeras, A.-L.Barra, and M.Potemski, How Perfect Can Graphene Be? Phys. Rev. Lett. 103, Article Number: 136403 (2009).

[21] C. Faugeras, M. Amado, P. Kossacki, M.Orlita, M. Kuehne, A.A. Nicolet, Yu.I. Latyshev, and M. Potemski, Magneto-Raman Scattering of Graphene on Graphite: Electron Scattering and Phonon Excitations. Phys. Rev. Lett. 107, Article Number: 036807 (2011).

[22] M. Kuhne, C. Faugeras, P. Kossacki, A.A. Nicolet, M. Orlita, Yu.I. Latyshev, and M. Potemski, Polarization-resolved magneto-Raman scattering of graphenelike domains on natural graphite. Phys. Rev. B. 85, Article Number: 195406 (2012)

[23] Yu. I. Latyshev, V.A. Bykov, A. V. Frolov, A.P. Orlov, Method of producing of atomic-thin single-crystallin films, Rus. Patent No. 2494037 (2013).

[24] A.V. Frolov, Yu.I. Latyshev, A.M. Smolovich, A.P. Orlov, Novel method of graphene films fabrication, Nonlinear World, No. 2, 138-139 (2013) (in Russian).

[25] R.R. Nair, P. Blake, A.N. Grigorenko, K.S. Novoselov, T.J.
Booth, T. Stauber, N.M. Peres, A.K. Geim, Fine structure constant defines visual transparency of graphene, Science 320, 1308-1308 (2008).

[26] E. S. Sadki, S. Ooi, and K. Hirata, Focused-ion-beam-induced deposition of superconducting nanowires, Applied Physics Letters, 85, 6206-6208 (2004).

[27] I.J. Luxmoore, I.M. Ross, A.G. Cullis, P.W. Fry, J. Orr, P.D. Buckle, J.H. Jefferson, Low temperature electrical characterisation of tungsten nano-wires fabricated by electron and ion beam induced chemical vapour deposition, Thin Solid Films 515, 6791-6797 (2007).

[28] E. Horvath, P.L. Neumann, A.L. Toth, Z.E. Horvatha, L.P. Biroa, Morphological and electrical study of FIB deposited amorphous W nanowires, Microelectronic Engineering 84, 837-840, (2007).

[29] W.X. Li, J.C. Fenton, Y.Q. Wang, D.W. McComb, P.A. Warburton, Tunability of the superconductivity of tungsten films grown by focused-ion-beam direct writing, Journal of Applied Physics 104, Article Number: 093913 (2008).

[30] Zhu Li-na, Li Guo-lu, Wang Hai-dou, Xu Bin-shi, Zhuang Da-ming, Liu Jia-jun, Microstructures and nano mechanical properties of the metal tungsten film, Current Applied Physics 9, 510-514 (2009).

[31] Quan Wang, Wei Mao, Daohan Ge, Yanmin Zhang, Ying Shao, and Naifei Ren, Effects of Ga ion-beam irradiation on monolayer graphene, Applied Physics Letters 103, Article number: 073501 (2013).

[32] Yu.I. Latyshev, A.M. Smolovich, A.P. Orlov, A.V. Frolov, V.S. Vlasenko. Proximity effect in periodic arrays of superconducting nanoislands on thin graphite layer. Abstracts for the 4th International scientific conference state-of-the-art trends of scientific research of artificial and natural nanoobjects (STRANN '14) 22-25, April 2014, Saint-Petersburg, Russia, 136-137. 